\title{
Hyperexpression of a-hemolysin explains enhanced virulence of sequence type 93 community-associated methicillin-resistant Staphylococcus aureus
}

Kyra YL Chua 1,2,3, , Ian R Monk', Ya-Hsun Lin' ${ }^{1}$, Torsten Seemann ${ }^{5}$, Kellie L Tuck ${ }^{6}$, Jessica L Porter ${ }^{1}$, Justin Stepnell', Geoffrey W Coombs ${ }^{7,8}$, John K Davies ${ }^{2}$, Timothy P Stinear ${ }^{1,2}$ and Benjamin P Howden ${ }^{1,2,3,4^{*}}$

\begin{abstract}
Background: The community-associated methicillin-resistant S. aureus (CA-MRSA) ST93 clone is becoming dominant in Australia and is clinically highly virulent. In addition, sepsis and skin infection models demonstrate that ST93 CA-MRSA is the most virulent global clone of $S$. aureus tested to date. While the determinants of virulence have been studied in other clones of CA-MRSA, the basis for hypervirulence in ST93 CA-MRSA has not been defined.

Results: Here, using a geographically and temporally dispersed collection of ST93 isolates we demonstrate that the ST93 population hyperexpresses key CA-MRSA exotoxins, in particular a-hemolysin, in comparison to other global clones. Gene deletion and complementation studies, and virulence comparisons in a murine skin infection model, showed unequivocally that increased expression of a-hemolysin is the key staphylococcal virulence determinant for this clone. Genome sequencing and comparative genomics of strains with divergent exotoxin profiles demonstrated that, like other $S$. aureus clones, the quorum sensing agr system is the master regulator of toxin expression and virulence in ST93 CA-MRSA. However, we also identified a previously uncharacterized AraC/XylS family regulator (AryK) that potentiates toxin expression and virulence in S. aureus.

Conclusions: These data demonstrate that hyperexpression of a-hemolysin mediates enhanced virulence in ST93 CA-MRSA, and additional control of exotoxin production, in particular a-hemolysin, mediated by regulatory systems other than agr have the potential to fine-tune virulence in CA-MRSA.
\end{abstract}

Keywords: Staphylococcus aureus, CA-MRSA, Pathogenesis, Alpha-hemolysin

\section{Background}

Community-associated methicillin-resistant Staphylococcus aureus (CA-MRSA) is an emerging global problem with very similar clinical presentations across different clones, despite significant genetic diversity [1]. Many CA-MRSA strains carry lukSF-PV in the accessory genome, which encodes the Panton-Valentine leukocidin (PVL), an exotoxin that causes neutrophil lysis [1]. Although there has been considerable controversy as to the role of this toxin in CA-

\footnotetext{
* Correspondence: benjamin.howden@austin.org.au

'Department of Microbiology and Immunology, University of Melbourne,

Melbourne, Victoria 3052, Australia

${ }^{2}$ Department of Microbiology, Monash University, Clayton, Victoria 3800,

Australia

Full list of author information is available at the end of the article
}

MRSA pathogenesis, some of this may be explained by a variable, species dependent susceptibility to PVL - human and rabbit neutrophils are lysed by PVL at very low concentrations whilst mouse and monkey neutrophils are less susceptible, making the interpretation of animal model data difficult in some cases [2]. Additionally, the importance of PVL is also likely to be dependent on the site of infection. In the rabbit pneumonia model, PVL has been demonstrated to have a clear role in mediating severe lung necrosis and inflammation [3]. In contrast, in skin infection, even in the rabbit model, its role remains less clear $[4,5]$.

Notwithstanding PVL, the increased expression of other core genome virulence determinants also contributes significantly to the increased virulence of CA-MRSA strains 
[6,7]. These include $\alpha$-hemolysin (Hla) and $\alpha$-type phenol soluble modulins (PSMs). Hla is a pore-forming exotoxin that lyses many cells including red cells, platelets, monocytes and endothelial cells [8]. Hla has been demonstrated to be an important mediator of virulence in skin infection and pneumonia $[9,10]$. The $\alpha$-type PSMs have been recently characterized and they lyse neutrophils and red cells $[11,12]$. The $\alpha$-type PSMs also mediate virulence in skin infection and septicemia and of these, PSM $\alpha 3$ is the most potent [11].

The study of unique, distantly related CA-MRSA clones that also demonstrate enhanced virulence, may provide insights into the emergence of the global CA-MRSA phenomenon, and also help define the genomic determinants of enhanced virulence. In Australia, the singleton ST93 CA-MRSA clone ST93 ("Queensland clone") has become dominant in the community [13], and we and others have demonstrated that our reference ST93 strain JKD6159 was highly virulent and caused severe skin infection in a mouse model compared to other CA-MRSA strains including USA300 [14,15].

In this study we used exotoxin analysis, functional genomics and a murine infection model to investigate the relative contribution of $\alpha$-hemolysin, $\alpha$-type phenol soluble modulins and Panton-Valentine leukocidin to the enhanced virulence of ST93 CA-MRSA. We show that increased virulence in the BALB/c mouse skin infection model is less dependent on $\alpha$-type phenol soluble modulin or PantonValentine leukocidin production but is instead due to highlevel expression of $\alpha$-hemolysin in this clone, controlled predominantly by the agr system.

\section{Results and discussion}

The emergence of CA-MRSA is a major public health issue, and there is a clear need to understand the basis for both virulence and transmission of global clones of CAMRSA. The genetically distinct CA-MRSA clone ST93-IV [2B] has rapidly become the dominant clone in Australia and its rise accounts for the increase in incidence of CAMRSA as a whole in this country [13]. We, and others have previously shown that ST93 strain JKD6159 is the most virulent global clone of $S$. aureus in murine models $[14,15]$. To determine the mediators of virulence in this clone we initially studied exotoxin expression in a large collection of ST93 S. aureus from around Australia, and compared representative high and low expressing strains to an international selection of clones.

\section{Exotoxin expression in ST93 CA-MRSA strains}

Staphylococcus aureus expresses a wide range of exotoxins that may contribute to virulence. Because Hla, PVL and $\alpha-$ type PSMs have been found by others to be important virulence factors in CA-MRSA strains $[9,11,16]$, we measured in vitro expression of these exotoxins by the wildtype ST93 strains and non-ST93 comparator strains. The main isolates used in this study are described in Table 1, while the collection of ST93 isolates from around Australia used for comparative exotoxin expression is from a study by Coombs et al. [17] and summarized in Additional file 1 . The comparison of expression of international clones to the ST93 reference strain JKD6159 and three additional ST93 strains selected for genome sequencing (see below) are shown in Figure 1, while the results for all 59 ST93 isolates compared to USA300 are shown in Additional file 2 ( $\alpha$-type PSMs) and Additional file 3 (Hla). The results of PVL analysis for the ST93 collection has been previously reported [17]. Because PVL is a 2-component exotoxin and both LukS-PV and LukF-PV are required for activity, we chose to measure LukF-PV expression by quantitative Western blot. LukF-PV was chosen over LukS-PV to obtain anti-LukF-PV antibody with increased specificity of binding as there was more sequence divergence between $l u k F-P V$ and the orthologous 2-component $S$. aureus exotoxins compared to $l u k S-P V$. Although there are four $\alpha$-type PSMs, PSM $\alpha 3$ causes the most significant neutrophil lysis [11] and we measured deformylated and $\mathrm{N}$-formylated PSM $\alpha 3$ expression by high performance liquid chromatography (HPLC). The proportion of deformylated and formylated forms of PSMs depends on the growth conditions, the activity of staphylococcal peptide deformylase and strain background $[7,18]$.

\section{PVL}

As previously reported [17], PVL expression was consistent across most ST93 strains. We found that there was no significant difference in the LukF-PV expression in the PVL positive strains JKD6159, TPS3104, USA300 and JKD6177. Although USA300 appeared to produce less LukF-PV than JKD6159, the difference was not statistically significant $(p=0.0943$, Figure 1A).

\section{PSMa3}

We found that the deformylated form of PSM $\alpha 3$ was almost always more abundant than the $\mathrm{N}$-formylated form (Figure 1B and Additional file 2). The ST30 CA-MRSA strain JKD6177 did not produce any PSM $\alpha 3$. There was no significant difference in PSM $\alpha 3$ expression between JKD6159 compared to USA300, however JKD6159 produced more PSM $\alpha 3$ compared to JKD6272 ( $\mathrm{p}=0.0003)$ and JKD6009 $(\mathrm{p}=0.0003)$. Compared to the other ST93 MRSA strains, JKD6159 produced more PSM $\alpha 3$ compared to TPS3105 ( $<0.0001)$, and TPS3106 $(\mathrm{p}=0.01)$ but less than TPS3104 ( $\mathrm{p}=0.0029)$ (Figure 1B). Expression levels across the whole ST93 collection were variable, although many isolates produced levels at least equivalent to USA300 (Additional file 2). 


\section{Table 1 Bacterial strains used in this study}

\begin{tabular}{|c|c|c|c|c|c|c|c|}
\hline S. aureus strain & Molecular type & Date of isolation & Place of isolation & Site of isolation & Relevant characteristics & lukSF-PV & reference \\
\hline \multicolumn{8}{|l|}{ Clinical isolates } \\
\hline JKD6159 & ST93-IV [2B] & 2004 & Victoria, Australia & Blood & Dominant Australian CA-MRSA clone & + & [14] \\
\hline TPS3104 & ST93-IV [2B] & 2009 & Western Australia, Australia & Nasal cavity & Dominant Australian CA-MRSA clone & + & This study \\
\hline TPS3105 & ST93-IV [2B] & 2005 & New South Wales, Australia & Blood & Australian CA-MRSA clone & - & This study \\
\hline TPS3106 & ST93-V [5C2\&5] & 2008 & Western Australia, Australia & Nasal cavity & Australian CA-MRSA clone & - & This study \\
\hline JKD6272 & ST1-IV [2B] & 2002 & Victoria, Australia & Blood & Australian CA-MRSA clone & - & [14] \\
\hline JKD6260 & ST1-IV [2B] & 2008 & Western Australia, Australia & Skin & Australian CA-MRSA clone & + & [14] \\
\hline JKD6177 & ST30-IV [2B] & 2003 & Melbourne, Australia & Blood & Australian CA-MRSA clone & + & [14] \\
\hline FPR3757 USA300 & ST8-IV [2B] & NA & San Francisco, USA & Wrist abscess & Dominant North American CA-MRSA clone & + & [19] \\
\hline JKD6009 & ST239-III [3A] & 2002 & New Zealand & Wound & Dominant Australian hospital-associated MRSA clone, AUS2/3 & - & {$[20]$} \\
\hline \multicolumn{8}{|l|}{ Mutant strains } \\
\hline JKD6159 $/$ lukSF-PV & ST93-IV [2B] & & & & Isogenic unmarked lukSF-PV KO of JKD6159 & - & This study \\
\hline 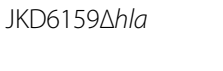 & ST93-IV [2B] & & & & $\begin{array}{l}\text { Isogenic unmarked hla KO of JKD6159. Deletion encompassed } \\
\text { genome coordinates } 1121291-1120441 \text {. }\end{array}$ & + & This study \\
\hline 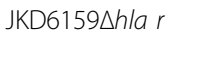 & ST93-IV [2B] & & & & 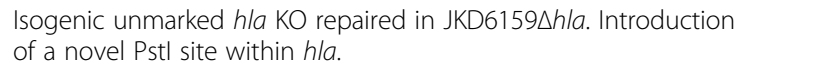 & + & This study \\
\hline JKD6159 $\Delta p s m a$ & ST93-IV [2B] & & & & $\begin{array}{l}\text { Isogenic unmarked psm-a KO in JKD6159. Deletion encompassed } \\
\text { genome coordinates 453364-45378. }\end{array}$ & + & This study \\
\hline JKD6159Apsmar & ST93-IV [2B] & & & & $\begin{array}{l}\text { Isogenic unmarked psm-a KO repaired in JKD6159 Dssm- } a \text {. Introduction } \\
\text { of a novel Sall site within psm-a }\end{array}$ & + & This study \\
\hline JKD6159 $\triangle 00043$ & ST93-IV [2B] & & & & $\begin{array}{l}\text { Isogenic unmarked SAA6159_00043 KO of JKD6159. Deletion encompassed } \\
\text { genome coordinates } 53156 \text { - } 54561\end{array}$ & + & This study \\
\hline JKD6159_AraCr & ST93-IV [2B] & & & & Isogenic AraC/XyIS regulator repaired in JKD6159 & + & This study \\
\hline TPS3105r & ST93-IV [2B] & & & & Isogenic agrA repair of TPS3105 & - & This study \\
\hline
\end{tabular}

KO: knockout, NA: not available. 


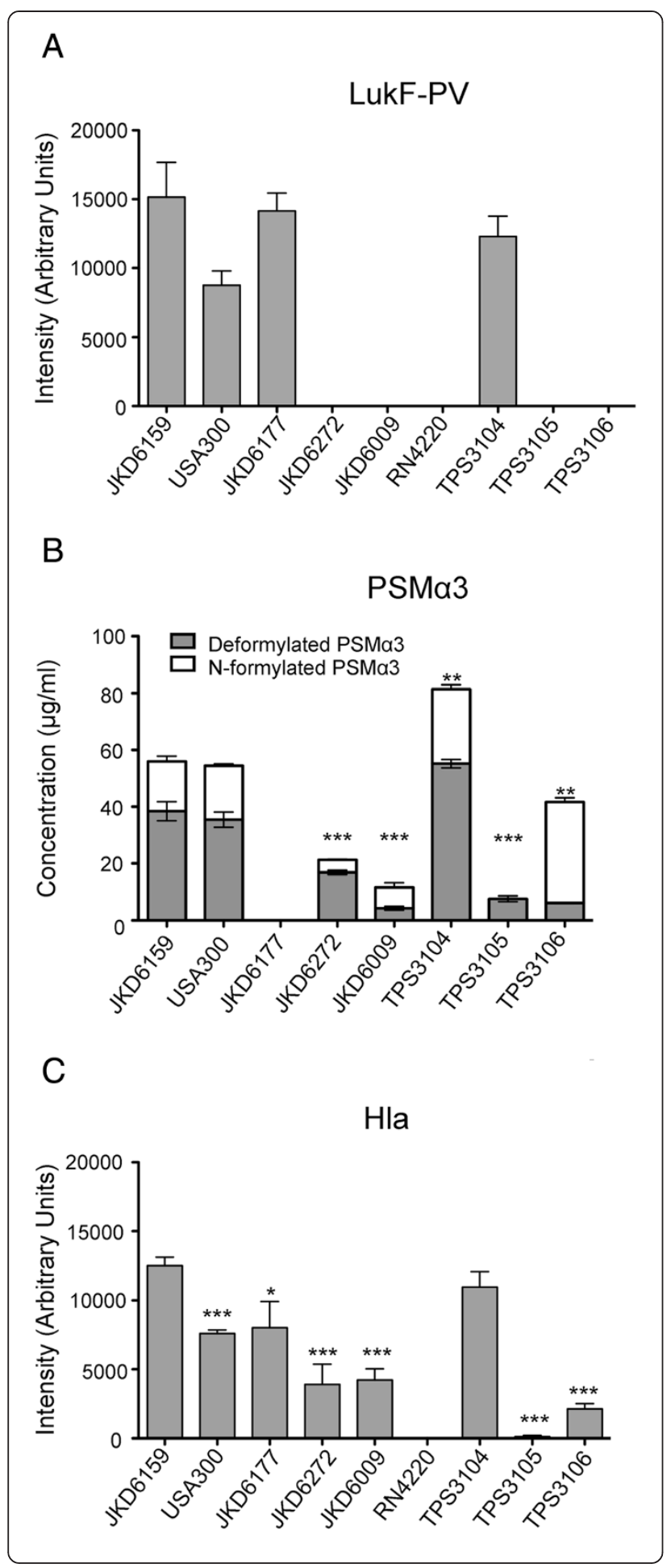

Figure 1 In vitro exotoxin expression of wildtype $S$. aureus isolates. JKD6159 (ST93-IV [2B]) compared with non-ST93 CA-MRSA strains FPR 3757 USA300 (ST8-IV [2B]), JKD6177 (ST30-IV [2B]), and JKD6272 (ST1-IV [2B]); Hospital-associated MRSA strain JKD6009 (ST239-III [3A]), wildtype ST93 strains TPS3104 (ST93-IV [2B]), TPS3105 (ST93-IV [2B]), and TPS3106 (ST93-V [5C2\&5]). (A) LukF-PV expression measured by quantitative Western blot. RN4220 was included as a negative control because it does not contain lukF-PV. All PVL negative strains did not express LukF-PV. There was no significant difference in the amount of LukF-PV expressed by the S. aureus strains containing IUKSF-PV. Data shown are mean intensity of bands in arbitrary units and SEM. (B) PSMa3 expression measured by HPLC. JKD6177 did not produce PSMa3. JKD6272 ( $p=0.0003)$, JKD6009 ( $p=0.0003)$, TPS3105 $(p<0.0001)$ and TPS3106 ( $p=0.0100)$ produced less deformylated and $\mathrm{N}$-formylated PSMa3 compared to JKD6159. There was no difference between PSMa3 production by JKD6159 and USA300. TPS3104 expressed more PSMa3 than JKD6159 ( $p=0.0029)$. Data shown are mean concentration $(\mu \mathrm{g} / \mathrm{ml})$, presented as vertical stacked bars and SEM. Deformylated PSMa3 is shown in grey bars. N-formylated PSMa3 is shown in white bars. (C) Hla expression measured by quantitative Western blot. RN4220 was included as a negative control because it does not express Hla. JKD6159 expressed more Hla compared to all non-ST93 wildtype strains ( $p<0.0001$ for all strains except JKD6177 $p=0.0107$ ). TPS3105 and TPS3106 produced significantly less Hla $(p<0.0001)$. There was no difference in Hla production between JKD6159 and TPS3104. Data shown are mean intensity of bands in arbitrary units and SEM. Note, ${ }^{* * *} p<0.001,{ }^{* *} p<0.01,{ }^{*} p<0.05$.

\section{Hla}

Hla expression appeared high for the majority of ST93 isolates, with the exception of four strains where expression was low (Additional file 3). JKD6159 produced greater levels of Hla than all the wildtype strains, including USA300 ( $p<0.0001$ for all strains except JKD6177, $\mathrm{p}=0.0107$, Figure $1 C$ ). There was no difference in Hla expression between JKD6159 and TPS3104.

Here we have demonstrated that the majority of ST93 strains consistently produce higher levels of Hla compared to other clones, including USA300, while production of PVL and $\alpha$-type PSM is similar, suggesting that enhanced expression of Hla may be responsible for increased virulence of ST93 CA-MRSA.

\section{Comparative virulence of ST93 isolates with differential exotoxin expression}

To further examine the role of these selected exotoxins in our mouse skin infection assay, we compared the virulence of four ST93 isolates selected based on their exotoxin expression profiles (high exotoxin expression, JKD6159 and TPS3104; low exotoxin expression, TPS3105 and TPS3106). TPS3104 was as virulent as JKD6159 in the mouse model in all outcome measures (Figure 2). In contrast, the strains with reduced exotoxin expression TPS3105 and TPS3106 were significantly less virulent compared to JKD6159, with less weight loss at day 5 of infection $(p<0.0001)$, smaller lesion size $(p<0.0001)$ and 

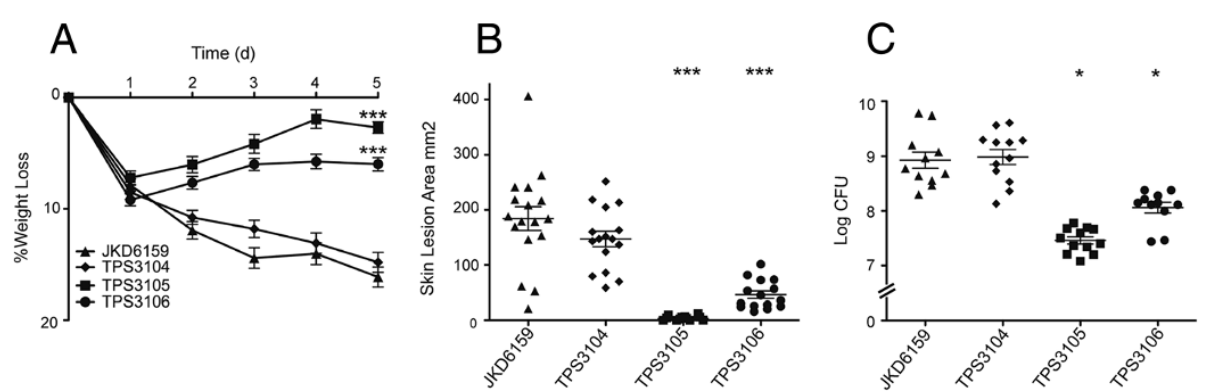

Figure 2 Virulence characteristics of wildtype ST93 CA-MRSA isolates. S. aureus JKD6159 compared with three other wildtype ST93 CA-MRSA isolates, TPS3104, TPS3105 and TPS3106 in a BALB/c mouse skin infection assay. At least 10 mice were used for each bacterial strain. (A) Weight loss induced by intradermal infection with S. aureus strains is demonstrated as percentage loss of weight over 5 days. The difference in percentage weight loss between JKD6159 and TPS3105 and TPS3106 was significant $(p<0.0001)$. There was no difference in weight loss between JKD6159 and TPS3104. Data shown are mean weight loss and SEM. (B) Skin lesion area $\left(\mathrm{mm}^{2}\right)$ at 5 days after infection was significantly greater with JKD6159 infected mice compared to TPS3105 and TPS3106 ( $p<0.0001)$. There was no difference in lesion area between JKD6159 and TPS3104. Data shown are mean area and SEM. (C) Recovery of S. aureus (log CFU) from infected tissues at 5 days after infection from JKD6159 infected mice was greater than with TPS3105 $(p=0.0177)$ and TPS3106 infected mice $(p=0.0328)$. There was no difference between JD6159 and TPS3104 infected mice. Data shown are mean CFU and SEM. Note, ${ }^{* *} p<0.001,{ }^{*} p<0.05$.

less CFU recovery from lesions (TPS3105, $\mathrm{p}=0.0177$; TPS3106, $\mathrm{p}=0.0328$ ) in the model (Figure 2).

\section{Impact of exotoxin expression on virulence of ST93 CA-MRSA in the murine skin infection model}

To further characterize the contribution of each of the exotoxins to disease in the murine model, genetic deletion and complementation experiments were performed for each of the selected toxins.

\section{Hla}

Given the increased in vitro expression of Hla by JKD6159 and TPS3104 and the apparent correlation of this increased expression with increased virulence in the mouse skin infection model, we generated JKD6159 4 hla and assessed this mutant in the mouse skin infection assay (Figure 3). There was a marked attenuation in virulence in all outcome measures with significantly decreased weight loss $(\mathrm{p}<0.0001)$, lesion size $(\mathrm{p}<0.0001)$ and CFU recovery $(\mathrm{p}=0.0177)$. To confirm that an unintentional mutation introduced during the procedure to knock-out hla was not responsible for the reduced virulence in this strain, complete genome sequencing of the strain using Ion Torrent sequencing was performed. Mapping of sequence reads from JKD6159 Ahla against JKD6159 (40× genome coverage) demonstrated no additional differences between JKD6159 and JKD6159Ahla. We also restored Hla function in this strain by using allelic replacement to repair the deletion in hla and create strain JKD61594hla $r$. As expected, Hla expression was absent in JKD6159 $4 h l a$ and expression was restored in JKD6159 4 hla $r$ when tested by Western Blot (Additional file 4A). JKD6159 4 hla $r$ also reverted to high virulence in the mouse skin infection assay (Figure 3). The apparent slight reduction in virulence of this hla repaired strain compared to wild type JKD6159 is explained by incomplete penetration of the restored hla allele in JKD6159 4 hla $r$, resulting in mixed bacterial populations and reversion to JKD6159 $\Delta$ hla for some of the mice (Additional file 4B and C).

\section{a-type PSMs}

In order to determine the contribution of $\alpha$-type PSMs to virulence of JKD6159, we generated JKD6159 $\Delta p s m \alpha$ (deletion of the whole $\alpha$-type PSM locus) and assessed this mutant in the mouse skin infection assay (Figure 3 ). There was no significant difference in virulence in all outcome measures; weight loss $(\mathrm{p}=0.06)$, lesion size $(\mathrm{p}=0.8174)$ and CFU recovery $(\mathrm{p}=0.1925)$. The absence of PSM $\alpha 3$ production was verified by HPLC and the integrity of the JKD6159 Apsma was confirmed by complementation (Additional file 5). It is noteworthy that the level of expression of PSM $\alpha 3$ by JKD6159 was similar to USA300 (Figure 1), a strain that produces high levels of PSMs and where a contribution to virulence has been demonstrated $[7,11]$. Despite this, the deletion mutant (JKD6159 $\Delta p s m \alpha$ ) demonstrated no attenuation of virulence compared to JKD6159 (Figure 3). The significantly divergent genetic background of ST93 compared with USA300 may account for this difference in the importance of $\alpha$-type PSMs to the virulence of JKD6159 [6].

PVL

We constructed an isogenic PVL negative mutant in JKD6159 by deleting lukSF-PV. Western Blot analysis confirmed the absence of LukF-PV in the mutant (Additional file 6). Assessment of the JKD6159 $l u k S F-P V$ mutant in the mouse skin infection model showed no decrease in virulence (Figure 3). Therefore PVL was not contributing to the increased virulence in JKD6159 in this 

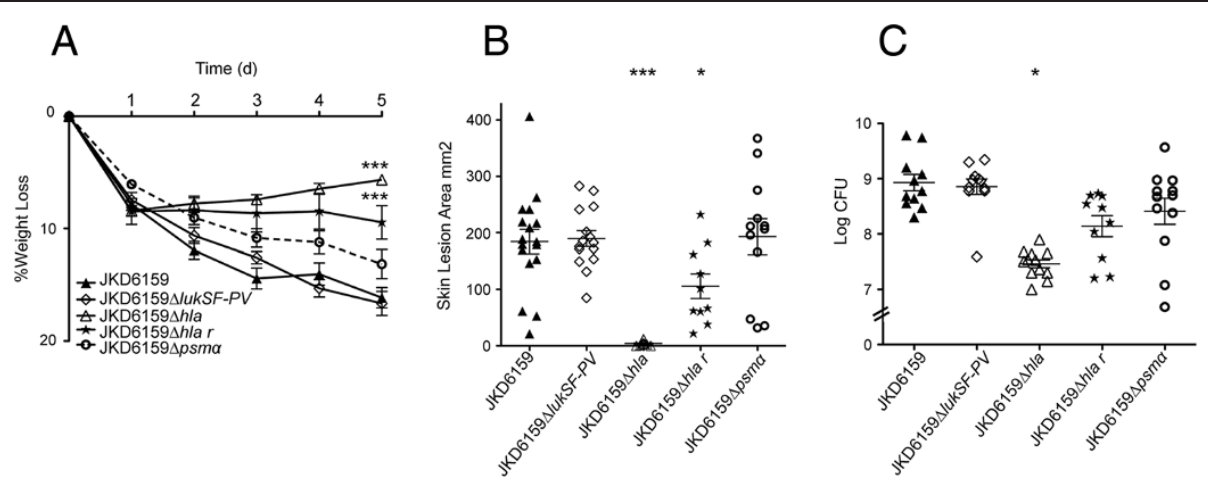

Figure 3 Virulence characteristics of S. aureus JKD6159 and isogenic exotoxin mutants derived from JKD6159. JKD6159 compared

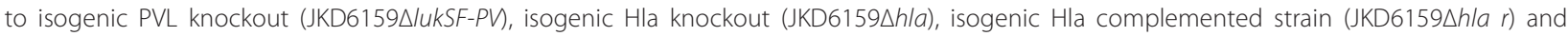
isogenic PSM-a knockout (JKD6159 $\mathrm{ppsma}$ ) in a BALB/C mouse skin infection assay. (A) Weight loss induced by intradermal infection with S. aureus strains is demonstrated as percentage loss of weight over 5 days. There was no significant difference between JKD6159,

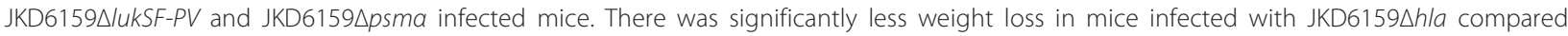

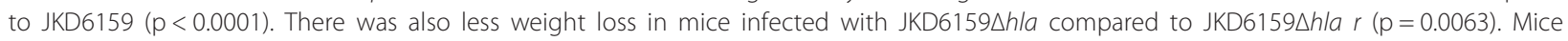
infected with JKD6159 $\Delta$ hla $r$ had less weight loss compared to JKD6159 $(p=0.0004)$. Data shown are mean weight loss and SEM. (B) There was no difference in skin lesion area $\left(\mathrm{mm}^{2}\right)$ at 5 days after infection in mice infected with JKD6159 and JKD6159D/uKSF-PV and

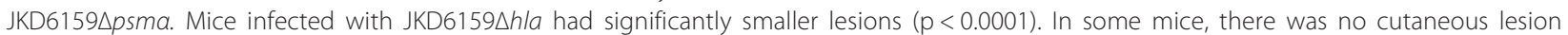

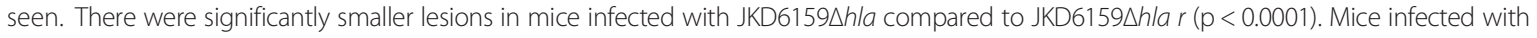
JKD6159 Ahla r had smaller lesions compared to JKD6159 ( $p=0.024)$. Data shown are mean area and SEM. (C) Recovery of S. aureus (log CFU)

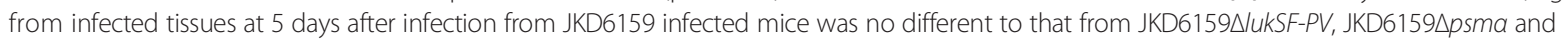

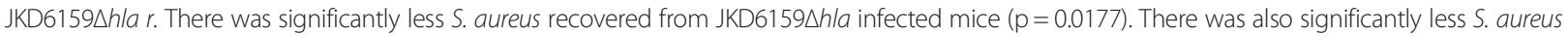

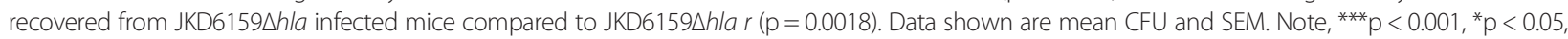
compared to JKD6159.

murine model. Murine neutrophils, unlike rabbit and human neutrophils are relatively resistant to the effects of PVL so it is difficult to draw firm conclusions as to the human importance of this result [2]. However, the aim of this study was to uncover the mechanisms for the observed increased virulence of ST93 previously demonstrated using this mouse model [14]. Our results reinforce the results of others who have examined different $S$. aureus clones which indicate that Hla, rather than PVL is the main mediator of virulence in CA-MRSA in a mouse skin infection model $[9,10,21,22]$. It should be noted that other authors have concluded that the rabbit skin infection model gave very similar results to the mouse model for infection at the same site [4]. Nonetheless, testing of our PVL deletion mutant in a rabbit model may be warranted in future.

\section{Genome sequencing of three additional ST93 isolates}

We have previously fully sequenced and annotated the genome of ST93 strain JKD6159 [14,23]. The differential virulence and exotoxin expression of some ST93 isolates compared to JKD6159 was then exploited by using whole genome sequencing and comparative genomics to determine the genetic basis for exotoxin expression in this clone. We selected the high expression strain TPS3104 and the low virulence and expression strains TPS3105 and TPS3106 to compare to JKD6159. De novo assembly of each of these strains resulted in $\sim 700$ contigs per isolate, with a genome length of $2.8 \mathrm{Mbp}$. The de novo assembly metrics are summarized in Additional file 7. The contigs were aligned to JKD6159 using BLASTN, with some important differences demonstrated between the strains (Figure 4A). TPS3104 contained SCCmecIV and фSA2 with lukSF-PV; TPS3105 contained SCCmecIV but lacked QSA2 and lukSF-PV; TPS3106 contained SCCmecV, and $S A 2$ without lukSF-PV. In addition, read-mapping against the complete JKD6159 genome (chromosome and plasmid: $2,832,164 \mathrm{bp}$ ) was then employed to define a $2,720,685$ bp core genome among these four isolates $(96 \%$ of the JKD6159 genome), revealing 253 polymorphic nucleotide positions, some of which were common to two strains. A phylogeny was inferred that confirmed the close relationship among all isolates, with TPS3106 more distantly related to the others (Figure 4B). The unmapped reads from each isolate were also subjected to de novo assembly to identify DNA not present in JKD6159. TPS3104 and TPS3105 contained no new sequences, while TPS3106 contained $34 \mathrm{~kb}$ of additional DNA, predominantly spanning the SCCmecV region.

\section{Comparative genomics of ST93 and the importance of agr} in the virulence of ST93 CA-MRSA

We next explored the contribution of specific mutations to the differential virulence of the ST93 strains. Using our read mapping approach described above, we compared the genome sequences of TPS3104, TPS3105 and TPS3106 


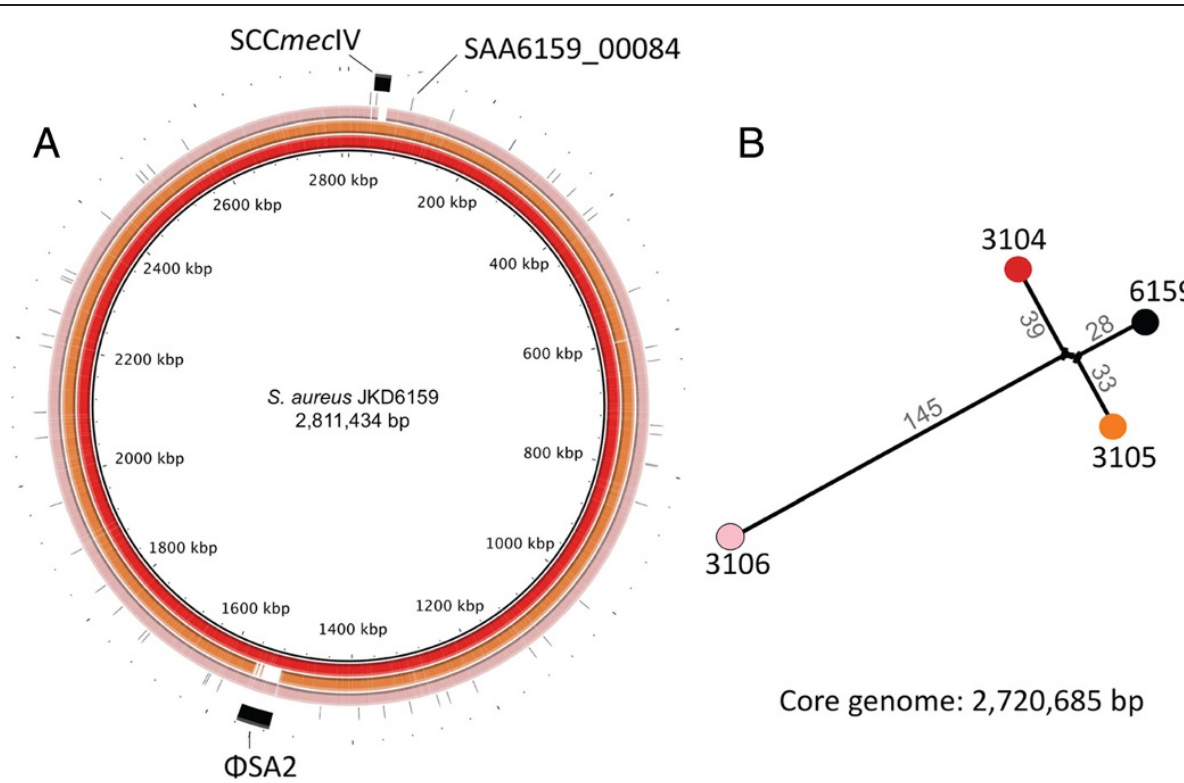

Figure 4 Whole genome sequence analysis and comparison of JKD6159 with other ST93 CA-MRSA isolates. (A) Circular diagram of the JKD6159, TPS3104, TPS3105 and TPS3106 chromosomes (from inner to outer circles). TPS3104, TPS3105 and TPS3106 contigs were mapped by BLASTN to JKD6159. TPS3104 contained SCCmecIV and \$SA2 with lukSF-PV; TPS3105 contained SCCmecIV but lacked $\phi S A 2$ and lukSF-PV; TPS3106 contained SCCmecV, and $\$ S A 2$ without lukSF-PV. (B) ST93 S. aureus phylogeny inferred by split decomposition analysis from pairwise comparisons of the 253 variable nucleotide positions identified from the ST93 core chromosome of 2,720,685 bp. Figures indicate the number of nucleotide substitutions per branch. All nodes have 100\% bootstrap support.

with each other and with JKD6159. There were a number of single nucleotide polymorphisms (SNPs) and insertions and deletions (indels) differentiating the strains from JKD6159 (Additional files 8, 9, 10). We searched for mutations in regulatory genes that could potentially explain the different virulence phenotypes of the strains. Notably, both avirulent ST93 strains, TPS3105 and TPS3106 contained mutations within the agr locus. We have since completed whole genome sequencing of TPS3151 and TPS3161 and found they contain predicted amino acid substitutions in AgrC that might disrupt agr function (Stinear et al., submitted). These isolates demonstrated low expression of Hla (Additional file 3).

Additionally, TPS3106 also contained a mutation in a gene encoding a previously uncharacterized AraC/ XylS family regulatory protein. This was also of particular interest as members of this class have been shown to contribute to the regulation of exotoxin expression $[24,25]$.

TPS3105 contained a frame-shift mutation within agrA (Sa_JKD6159 nucleotide 2096502) and a further substitution ( $\mathrm{G}$ to A) within agrA at nucleotide 2096569), while TPS3106 contained an $~ 356$ bp deletion spanning the agr effector molecule, RNAIII (deletion spanning nucleotides 2093372 to 2093728). These mutations suggested these isolates were agr deficient. To demonstrate that these changes explained the reduced exotoxin expression and loss of virulence in TPS3105 we repaired
agrA using allelic exchange to create TPS3105r (the parental strain TPS3105 now containing an intact agrA). Quantitative real time RT-PCR for RNAIII demonstrated that TPS3105r produced 325-fold more RNAIII than TPS3105. Virulence was also restored and TPS3105r caused greater weight loss, skin lesion area and CFU recovery from lesions compared to the parental strain TPS3105 ( $<0.0001$, Figure 5). There was no significant difference between JKD6159 and TPS3105 $r$ in all outcome measures in the mouse skin infection model (Figure 5). These experiments show that intact agr is essential for the virulence of ST93 CA-MRSA. The agrA repaired mutant of TPS3105, TPS3105 $r$ expressed significantly greater amounts of PSM $\alpha 3(\mathrm{p}<0.0001)$ and Hla $(\mathrm{p}=$ 0.0019), consistent with agr control of these virulence determinants (Figure 6). Thus, despite the genetic divergence of ST93 from other S. aureus [14], the molecular foundation of virulence for this CA-MRSA clone is similar in this respect to USA300 $[9,26,27]$ and other $S$. aureus strains $[28,29]$, where the importance of agr has been very well established.

\section{The AraC/XylS regulator (AryK) enhanced Hla expression} and virulence in ST93 CA-MRSA

The SNP at position 92551 in SAA6159_00084 introduced a premature stop codon and created a pseudogene within SAA6159_00084 in JDK6159, however the gene was intact in TPS3106. The intact version of this gene, which was also 

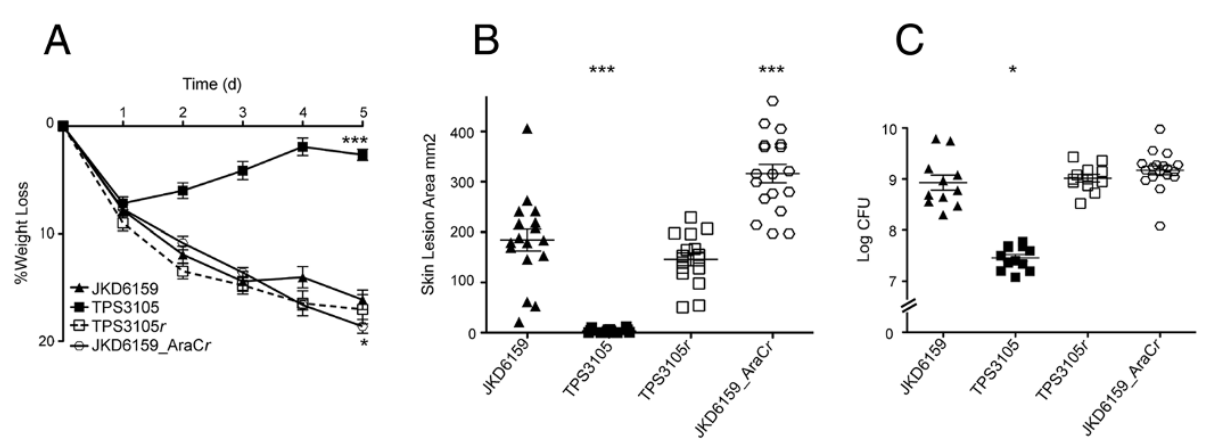

Figure 5 The importance of agr and aryK in the virulence of ST93 CA-MRSA. Isogenic repaired agr mutant TPS3105r compared to TPS3105 and JKD6159, and JKD6159 compared with isogenic repaired AraC/XylS family regulator mutant (JKD6159_AraCr) in a BALB/C mouse skin infection assay. At least 10 mice were used for each bacterial strain. (A) Weight loss induced by intradermal infection with $\mathrm{S}$. aureus strains is demonstrated as percentage loss of weight over 5 days. There was no difference between JKD6159 and TPS3105r in all outcome measures. TPS3105 $r$ infected mice had significantly increased weight loss compared to TPS3105 ( $p<0.0001)$. There was a small increase in weight loss in mice infected with JKD6159_AraCr compared to JKD6159 $(\mathrm{p}=0.0311)$. Data shown are mean weight loss and SEM. (B) Skin lesion area $\left(\mathrm{mm}^{2}\right)$ at 5 days after infection in TPS3105r infected mice was significantly increased compared to TPS3105 ( $p<0.0001)$. Mice infected with JKD6159_AraCr had increased lesion area compared with JKD6159 ( $p<0.0001$ ). Data shown are mean area and SEM. (C) Recovery of S. aureus (log CFU) from infected tissues at 5 days after infection from TPS3105r was significantly greater than from TPS 3105 infected mice $(p<0.0001)$. There was no difference in S. aureus recovered from mice infected with JKD6159 and JKD6159_AraCr. Data shown are mean CFU and SEM. Note, ${ }^{* * *} p<0.001,{ }^{*} p<0.05$.

intact in 19 other publically available $S$. aureus genome sequences we examined, encodes a previously uncharacterized AraC/XylS family regulatory protein. While the virulence attenuation in TPS3106 was likely a direct result of the agr deficiency, we also wanted to determine if the novel regulator mutation in SAA6159_00084 impacted the virulence in ST93 S. aureus.

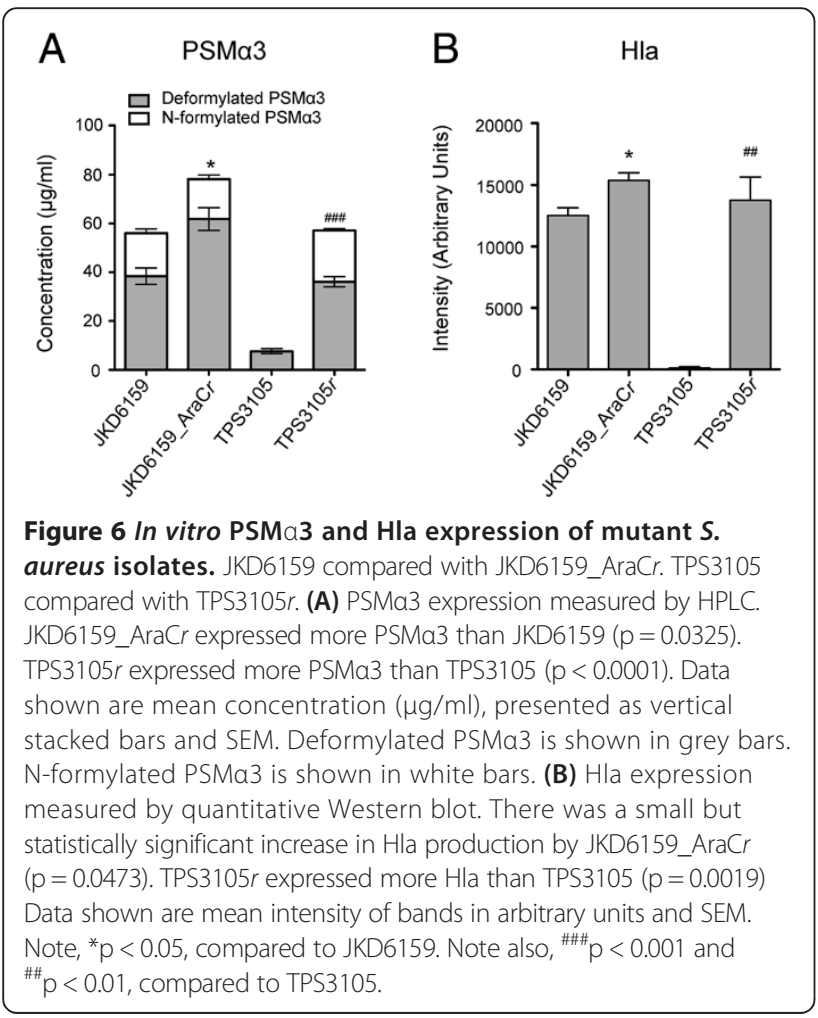

To test the hypothesis that SAA6159_00084 encoded a regulator of virulence, we repaired the premature stop codon in SAA6159_00084 in JKD6159 using allelic exchange to generate strain JKD6159_AraCr. To confirm we had not introduced additional DNA changes during allelic exchange we sequenced the whole genome of JKD6159_AraCr and found no additional mutations (35x coverage). JKD6159_AraCr encoding an intact copy of SAA6159_00084 demonstrated a modest, but significant increase in virulence as indicated by lesion size $(\mathrm{p}<$ 0.0001 ) and weight loss in the mouse skin infection assay $(\mathrm{p}=0.0311$, Figure 5$)$, suggesting that this protein is a positive regulator of virulence in CA-MRSA strains. JKD6159_AraCr expressed more PSM $\alpha 3$ ( $\mathrm{p}=$ $0.0325)$ and Hla $(\mathrm{p}=0.0473)$ than its parental strain JKD6159 that was consistent with an increase mouse skin lesion size (Figure 6). We propose the name aryK for SAA6159_00084 (AraC family-like gene).

\section{RNAseq demonstrates global regulatory impact of AryK}

To investigate the regulatory impact of AryK, RNAseq was performed using RNA extracted from stationary phase cultures (Figure 7). This growth phase was selected as we reasoned that AryK might be interacting with $a g r$ and thus any impacts on Hla expression would be greatest at this time. A small number of virulenceassociated loci were down regulated in the $\operatorname{aryK}$ mutant (JKD6159), including beta-type phenol soluble modulins (SAA6159_01024 and SAA6159_01025), and the virulence regulator saeS. However, the most dramatic and significant transcriptional changes were found in genes involved in central metabolic functions. Using the Kyoto Encyclopedia of Genes and Genomes (KEGG) pathway 


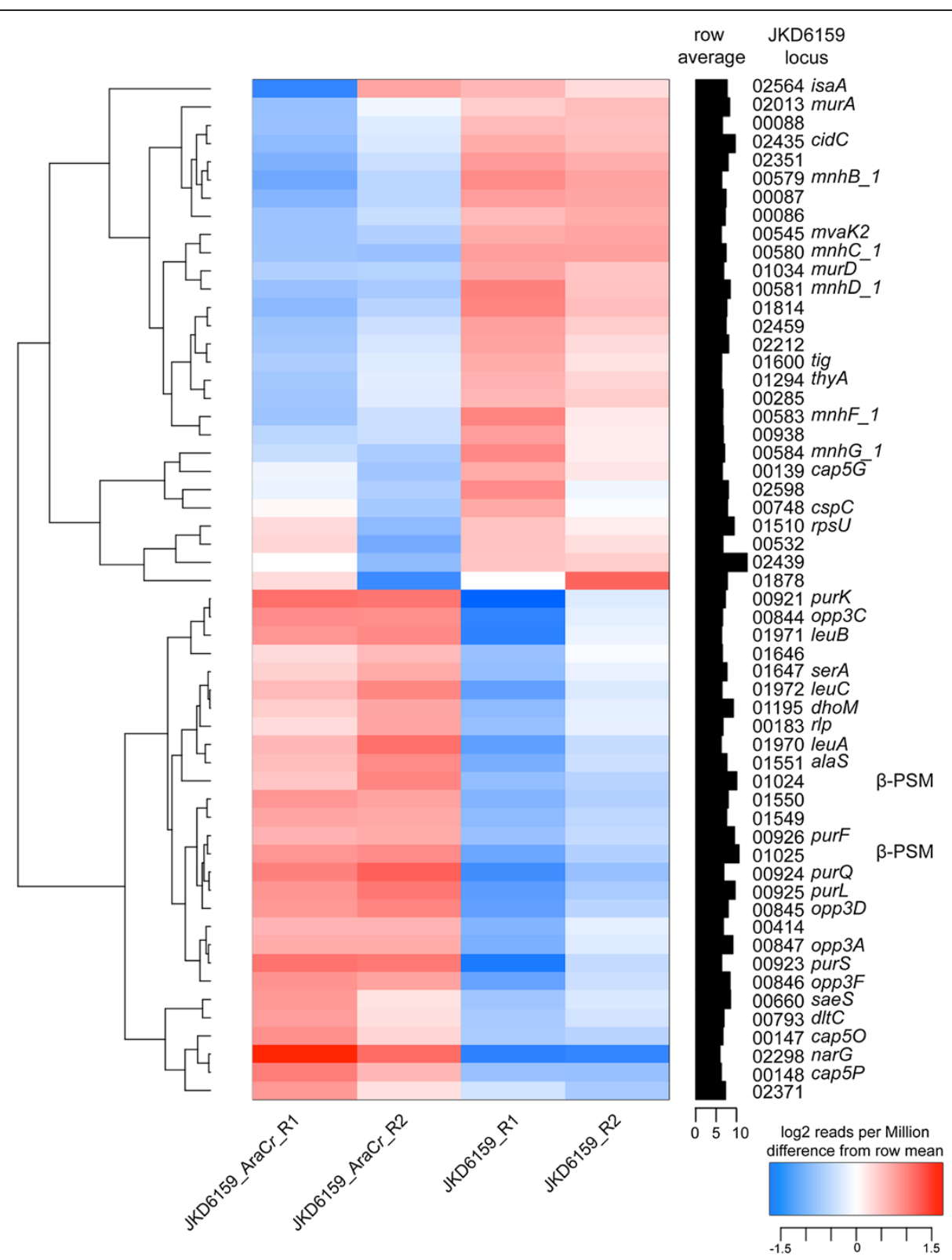

Figure 7 Heatmap of RNA Sequencing comparing JKD6159 (aryK inactive) to JKD6159_AraCr (aryK intact). RNA seq was performed in duplicate from stationary phase cultures. This heatmap, clustered on expression profiles, was created based on $\log _{2}$ transformed counts to identify consistent changes in expression profiles between strains. To be included in the heat map, genes were required to have at least 1000 counts (reads), totaled over all samples, where the standard deviation of $\log _{2}$ expression differences had to exceed two. The heatmap highlights significant aryK-dependent changes, in particular genes involved in the regulation of central metabolic functions.

analysis (www.genome.jp) [30] the major pathways where differential transcription occurred were down regulation of genes linked to purine metabolism (purK, purS, purQ, purL, purF), valine, leucine and isoleucine biosynthesis (leuA, leuB, leuC), and oligopeptide transport (орp3C, opp $3 D$, opp $3 F$, opp $3 A$ ). In contrast up regulation of genes encoding cation transport systems (mnhB_1, mnhC_1, mnhD_1, mnhF_1, mnhG_1) was found.

Here, we have clearly demonstrated that $a g r$ is the major "on-off" switch for virulence in ST93 CA-MRSA, but we also found that other genetic changes are impacting virulence gene regulation in a clone-specific manner. We speculate that the inactivation of aryK may have been an evolutionary response by ST93 CA-MRSA to modulate or fine-tune the amount of Hla and other factors required for host persistence. There are six AraC/ XylS family regulators in S. aureus (SA0097, SA0215, SA0622, SA1337, SA2092, SA2169; S. aureus strain N315 locus tags). Two of these, Rbf (SA0622) and Rsp (SA2169) have been studied and demonstrated in other $S$. aureus 
strains to regulate biofilm formation and modulate expression of surface-associated proteins [24,25,31]. In contrast, we found that $\operatorname{ary} K$ increases Hla expression and virulence, acting as a positive regulator of virulence by directly or indirectly upregulating exotoxin expression, without an apparent effect on agr expression in stationary phase.

\section{Conclusions}

In this study, we have obtained insights into the genetic basis for the increased virulence of ST93 by using a combination of comparative and functional genomics. We have demonstrated the key role of Hla and agr and shown how an additional novel regulatory gene, aryK by a loss-of-function point mutation, is modulating virulence in this clone. Quantification of exotoxin expression in a larger collection of ST93 strains demonstrated that the findings in strain JKD6159 are relevant to the majority of the ST93 population isolated from around Australia as exotoxin expression in JKD6159 is representative of most of the ST93 population. Our study highlights the power of comparative genomics to uncover new regulators of virulence but it also shows the complex nature of these changes even in closely related bacterial populations. Careful strain selection, detailed comparative genomics analyses, and functional genomic studies by creating multiple genetic changes in one strain will be required to gain a full insight into the genetic basis for the emergence and hypervirulence of ST93 CA-MRSA.

\section{Methods}

\section{Ethics statement}

This study was performed in accordance with the Australian Prevention of Cruelty to Animals Act 1986 and the Australian code of practice for the care and use of animals for scientific purposes. The protocol was approved by the Animal Ethics Committee of the University of Melbourne (Permit Number: 0911248.2).

\section{Bacterial strains and culture}

Bacterial strains used in this study are summarized in Table 1 (international clone collection) and Additional file 1 (ST93 strain collection), and include the ST93 reference strains JKD6159, USA300 strain FPR3757 [19], ST30 strain JKD6177, and the HA-MRSA ST239 clone JDK6009 [20], as well as 58 additional ST93 collected from around Australia and previously reported [17]. For all experiments except exotoxin expression bacteria were grown in brain heart infusion broth (BHI, Oxoid). For the mouse skin infection assay, S. aureus were harvested at the stationary phase of growth after 18 hours incubation $\left(\mathrm{OD}_{600} \sim 2.0\right)$, washed, diluted and resuspended in PBS. The bacterial inoculum (CFU) and viable counts were determined by plating onto BHI agar and colony enumeration.

For LukF-PV expression experiments, bacteria were grown in CCY media (3\% yeast extract (Oxoid), 2\% Bacto Casamino Acids (Difco), 2.3\% sodium pyruvate (Sigma-Aldrich), 0.63\% $\mathrm{Na}_{2} \mathrm{HPO}_{4}, 0.041 \% \mathrm{KH}_{2} \mathrm{PO}_{4}, \mathrm{pH}$ 6.7). For $\alpha$-hemolysin (Hla) and PSM $\alpha 3$ expression experiments, bacteria were grown in tryptone soy broth (TSB, Oxoid). Overnight cultures were diluted 1:100 into fresh media and then incubated at $37^{\circ} \mathrm{C}$ with shaking $(180 \mathrm{rpm})$ until stationary phase was achieved. For LukF-PV detection, isolates were cultured for 8 hours $\left(\mathrm{OD}_{600} \sim 1.8\right)$; for Hla detection, isolates were cultured for approximately 3 hours $\left(\mathrm{OD}_{600} \sim 1.8\right)$; and for PSM $\alpha 3$ detection, isolates were cultured for 24 hours $\left(\mathrm{OD}_{600} \sim 2.0\right)$. Culture supernatants were harvested by centrifugation and filter sterilized. These were performed in at least triplicate for each $S$. aureus strain.

\section{Detection of LukF-PV and Hla by western blotting}

Trichloroacetic acid was added to culture supernatants and incubated at $4^{\circ} \mathrm{C}$ overnight. Precipitates were then harvested by centrifugation, washed with acetone, airdried and solubilized in a SDS and 2-mercaptoethanol containing sample buffer. The proteins were separated on 12\% SDS-PAGE. A peptide sequence specific to LukF-PV, HWIGNNYKDENRATHT was synthesized and HRP conjugated polyclonal chicken IgY was raised against this peptide (Genscript). This antibody was used to detect LukF-PV with enhanced chemiluminescence. Images generated from the western blots were quantitated using GS800 Calibrated Densitometer (BioRad) and Image J [32].

Hla was detected using a polyclonal rabbit anti-Hla (Sigma-Aldrich), in buffer containing $20 \mathrm{mM} \mathrm{DEPC} \mathrm{to}$ inhibit non-specific protein A binding and HRP conjugated sheep anti-rabbit secondary antibody (Millipore) with enhanced chemiluminescence detection [33]. For comparison of JKD6159 versus international clone collection (Table 1), images generated from the Hla western blots were quantitated using GS800 Calibrated Densitometer (BioRad) and Image J [32].

Subsequently, for comparison of JKD6159 and other ST93 strains (Table 1), detection of chemiluminescence was performed using the MF-ChemiBIS 3.2 platform (DNR Bioimaging systems). Quantitation was performed using Image $\mathrm{J}$ [32].

\section{Detection of PSMa3 expression}

HPLC chromatography was performed on an Agilent Technology 1200 Series system with an analytical Agilent Eclipse XDB-C18 $(4.6 \mathrm{~mm} \times 150 \mathrm{~mm})$ column. A water/ acetonitrile gradient $(0.1 \%$ trifluoroacetic acid) from $0-$ $100 \%$ acetonitrile over $28 \mathrm{~min}$ at a flow rate of $1 \mathrm{~mL} / \mathrm{min}$ 
was used. The total run time was $32 \mathrm{~min}$, and peaks were quantified at a wavelength of $214 \mathrm{~nm}$. The deformylated and formylated form of PSM $\alpha 3$ MEFVAKLFKFFKDLL GKFLGNN was identified in the $S$. aureus TSB culture supernatants by comparison of their retention times to a commercially synthesized PSM $\alpha 3$ standard (GenScript) and by spiking the samples with the synthesized standards. The identity of the deformylated peptide present in the samples was confirmed by analysing collected fractions by ESI-MS. There was only one peptide present in this fraction; the deformylated form of PSM $\alpha 3$. In contrast, other peptides were observed in the fractions of USA300, JKD6272, TPS3104, TPS3105r, and JKD6159_AraCr containing the $\mathrm{N}$-formylated form of PSM $\alpha 3$. In these cases, the percentage of $\mathrm{N}$-formylated PSM $\alpha 3$ peptide was determined using the total ion count of the major peaks in the ESI-MS and the peak area of the HPLC chromatogram was adjusted accordingly. The concentrations of the deformylated and formylated forms of PSM $\alpha 3$ were determined by comparison of their peak areas to those of the synthesized standards. The standard curves were constructed in the concentration range of $6.2-100 \mu \mathrm{g} / \mathrm{mL}$ and were linear over this range.

\section{DNA methods, molecular techniques and construction of mutants}

DNA was extracted using the GenElute kit according to the manufacturer's instructions (Sigma-Aldrich). A lukSF-PV knockout, hla knockout and a repaired agrA of TPS3105 were generated according to the published method [34]. For the knockouts, flanking sequences were amplified and ligated prior to cloning with pKOR1. For allelic replacement to generate TPS3105r, a PCR product of agrA from JKD6159 was cloned with pKOR1. For allelic replacement JKD6159_AraCr, a PCR product of this AraC regulator from TPS3106 was cloned with pKOR1. The deletion of the whole psm $\alpha$ locus in JKD6159,

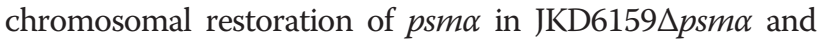
the restoration of Hla expression in JKD6159 4 hla were conducted using the pIMAY protocol described by Monk et al. [35]. Knockout and restoration amplimers were cloned into pIMAY by SLIC [36]. The primers used are listed in Additional file 11. The knockout and restoration clones were confirmed by PCR and Sanger sequencing of the mutated locus. Mutations were further validated with functional assays of activity, which included sheep blood hemolysis, western blot, and/or HPLC.

\section{Mouse skin infection assay}

Mice were infected with $S$. aureus as previously described [14]. Briefly, six-week-old female BALB/c mice were infected by intradermal injection with $10^{8} \mathrm{CFU}$ of S. aureus. Mice were assessed and weighed daily for five days. Mice were culled on the 5th day and lesion size measured and CFU recovered from infected tissues by homogenization and colony enumeration on BHI. For each $S$. aureus strain, at least 10 mice were assessed.

\section{Genome sequencing}

Genome sequences for three ST93 strains (TPS3104, TPS3105, TPS3106) were obtained from an Illumina GAIIx analyzer using 100 bp paired-end chemistry with a mean fold coverage of 331×. Genome sequencing of the two laboratory-induced mutants JKD6159 Ahla (TPS3265) and JKD6159_AraCr (TPS3268) was performed using Ion Torrent sequencing technology.

\section{Comparative genomics}

A read mapping approach was used to compare the sequences from all isolates used in this study, as previously described [14,37]. Briefly, the reads from all genomes were aligned to the JKD6159 reference using SHRiMP 2.0 [38]. SNPs were identified using Nesoni v0.60 [www. bioinformatics.net.au]. Using the whole genome sequence of JKD6159 as a reference, a global SNP analysis was performed, and allelic variability at any nucleotide position was tallied to generate a global SNP analysis for every genome compared to JKD6159.

\section{Quantitative RT-PCR for RNAIII expression}

To investigate activity of the agr locus (RNAIII) qRT-PCR was performed for RNAIII as previously described [37]. Briefly, RNA was prepared as previously described with two on-column DNase I digestion steps and cDNA synthesis using SuperScript II reverse transcriptase (Invitrogen). Relative expression was determined as previously described and was normalised against gyrB. Results were obtained from 3 biological replicates each performed in triplicate.

\section{RNA sequencing}

Staphylococcus aureus strains JKD6159 and JKD6159_Ar$\mathrm{aCr}$ were grown to early stationary culture as described above. For RNA protection, 0.5 volumes of RNAlater RNA stabilization reagent (Qiagen) was added immediately to the liquid culture and allowed to incubate with the bacterial suspension for 15 minutes at room temperature. Cells were pelleted at $5,000 \times \mathrm{g}$ for 5 minutes followed by RNA extraction using RNeasy mini kit (Qiagen) and two rounds of DNase I digestion (Qiagen) according to the manufacturer's instruction. RNA concentration was quantified using Qubit ${ }^{\oplus}$ 2.0 Fluorometer and RNA quality assessed using Agilent 2100 Bioanalyzer. Ten $\mu \mathrm{g}$ of total RNA from the stationary growth phase with RNA intergrity number (RIN) greater than 7 was used in RNA-seq. Ribosomal depletion, cDNA library preparation and pair ended sequencing using HiSeq2000 sequencing platform was performed by Beijing Genome Institute 
(Hong Kong, China). RNAseq was performed on two biological samples for each strain.

RNAseq reads were mapped onto the JKD6159 reference genome [23], using SHRiMP 2.2.2 [39]. Alignment to CDS features from each biological replicate of each strain provided counts that were a measure of mRNA levels. Counts were normalized using the trimmed-mean normalization function in edgeR, part of the BioConductor package [40]. A heat map was created based on $\log _{2}$ transformed counts to identify consistent changes in expression profiles between strains. To be included in the heat map, genes were required to have at least 1000 counts, totaled over all samples, where and the standard deviation of the $\log _{2}$ expression levels had to exceed two.

\section{Statistical analysis}

Percentage mouse weight change at day 5 , viable counts of $S$. aureus in mouse tissues and skin lesion area of each isolate, Hla, LukF-PV and PSM $\alpha 3$ expression versus JKD6159 were analyzed using an unpaired $t$ test. A similar analysis was used to analyze virulence outcome measures and exotoxin expression between TPS3105 and TPS3105r. (There was no difference in results when Bonferonni analysis was performed). All analyses were performed using Prism 5 for Macintosh v5.0b (GraphPad Software Inc.).

\section{Availability of supporting data}

The data sets supporting the results of this article are in the NCBI Sequence Read Archive under study accession SRP004474.2 and the NCBI BioProject Archive under study accession PRJNA217697.

\section{Additional files}

\section{Additional file 1: Staphylococcus aureus ST93 strains used in} this study.

Additional file 2: Expression of PSMa3 by ST93 strains and USA300. (A) Expression of deformylated PSMa3. (B) Expression of $\mathrm{N}$-formylated PSMa3. Data shown are mean concentration $(\mu \mathrm{g} / \mathrm{ml})$ and SEM.

Additional file 3: Expression of Hla by ST93 strains and USA300. Hla expression measured by quantitative Western blot. Data shown are mean intensity of bands in arbitrary units and SEM.

Additional file 4: Hla Western Blot of JKD6159, JKD6159 $h$ hla and

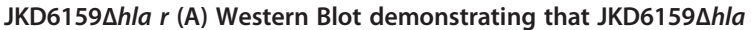
does not express Hla by Western Blot and that complementation of this mutant (JKD6159 $\mathrm{hla} r$ ) results in restoration of Hla expression. (B) Arrangement of PCR primers used PCR screen of JKD6159 $\triangle$ hla and JKD6159 4 hla r. (C) PCR screen of 25 randomly selected S. aureus colonies obtained from two mice (mouse 4 and mouse 7 ) post skin infection with JKD6159 4 hla $r$. The PCR primers used flank the region deleted in hla for the mutant and show incomplete penetration of the bacterial population with the repaired version of hla (17/25 with an intact allele for mouse 4 and $21 / 25$ for mouse 7 ), thereby explaining the inability of the repaired mutant to fully restore the virulence phenotype in this infection model.

Additional file 5: Detection of formylated PSMa3 in JKD6159, JKD6159 JKD6159 $\Delta$ sma did not produce formylated PSMa3. Complementation of this strain resulted in restoration of formylated PSMa3 expression. In all strains $\delta$-toxin expression was maintained.

Additional file 6: LukF-PV Western Blot of JKD6159 and

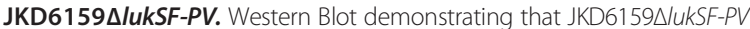
does not express LukF-PV

Additional file 7: Table of de novo assembly characteristics for $S$. aureus strains TPS3104, TPS3105 and TPS3106.

Additional file 8: Table of single nucleotide differences between JKD6159 and TPS3104.

Additional file 9: Table of single nucleotide differences between JKD6159 and TPS3105.

Additional file 10: Table of single nucleotide differences between JKD6159 and TPS3106.

Additional file 11: Table of primers used in this study.

Competing interest

No author has any competing interests to declare.

\section{Authors' contributions}

Conceived the project, TPS, BPH, KYLC, JKD; performed the experiments, KYLC, IRM, YHL, JLP, GWC, JS, KLT; analysed the data, KYLC, YHL, TPS, BPH, TS, $\mathrm{KLT}$; wrote the manuscript, KYLC, BPH, TPS. All authors read and approved the final manuscript.

Authors' information

Timothy P. Stinear and Benjamin P. Howden are the Joint Senior Authors.

\section{Acknowledgements}

We thank Kirstie Mangas and Brian Howden for expert technical assistance.

\section{Author details}

${ }^{1}$ Department of Microbiology and Immunology, University of Melbourne, Melbourne, Victoria 3052, Australia. ${ }^{2}$ Department of Microbiology, Monash University, Clayton, Victoria 3800, Australia. ${ }^{3}$ Austin Centre for Infection Research (ACIR), Infectious Diseases Department, Austin Health, PO Box 5555, Heidelberg, Victoria 3084, Australia. ${ }^{4}$ Microbiology Department, Austin Health, Heidelberg, Victoria 3084, Australia. ${ }^{5}$ Victorian Bioinformatics Consortium, Monash University, Clayton, Victoria 3800, Australia. 'School of Chemistry, Monash University, Clayton, Victoria 3800, Australia. ${ }^{7}$ Australian Collaborating Centre for Enterococcus and Staphylococcus Species (ACCESS) Typing and Research, PathWest Laboratory Medicine-WA, Royal Perth Hospital, Perth, Western Australia 6000, Australia. ${ }^{8}$ School of Biomedical Sciences, Curtin University, Bentley, Western Australia 6102, Australia.

Received: 6 December 2013 Accepted: 5 February 2014 Published: 10 February 2014

\section{References}

1. David MZ, Daum RS: Community-associated methicillin-resistant Staphylococcus aureus: epidemiology and clinical consequences of an emerging epidemic. Clin Microbiol Rev 2010, 23(3):616-687.

2. Loffler B, Hussain M, Grundmeier M, Bruck M, Holzinger D, Varga G, Roth J, Kahl BC, Proctor RA, Peters G: Staphylococcus aureus Panton-Valentine leukocidin is a very potent cytotoxic factor for human neutrophils. PLoS Pathog 2010, 6(1):e1000715.

3. Diep BA, Chan L, Tattevin P, Kajikawa O, Martin TR, Basuino L, Mai TT, Marbach H, Braughton KR, Whitney AR, et al: Polymorphonuclear leukocytes mediate Staphylococcus aureus Panton-Valentine leukocidin-induced lung inflammation and injury. Proc Natl Acad Sci U S A 2010, 107(12):5587-5592.

4. Kobayashi SD, Malachowa N, Whitney AR, Braughton KR, Gardner DJ, Long D, Bubeck Wardenburg J, Schneewind O, Otto M, Deleo FR: Comparative analysis of USA300 virulence determinants in a rabbit model of skin and soft tissue infection. J Infect Dis 2011, 204(6):937-941.

5. Lipinska U, Hermans K, Meulemans L, Dumitrescu O, Badiou C, Duchateau L, Haesebrouck F, Etienne J, Lina G: Panton-Valentine leukocidin does play a role in the early stage of Staphylococcus aureus skin infections: a rabbit model. PLoS One 2011, 6(8):e22864. 
6. Li M, Diep BA, Villaruz AE, Braughton KR, Jiang X, DeLeo FR, Chambers HF, LU Y, Otto M: Evolution of virulence in epidemic community-associated methicillin-resistant Staphylococcus aureus. Proc Natl Acad Sci U S A 2009, 106(14):5883-5888.

7. Li M, Cheung GY, Hu J, Wang D, Joo HS, Deleo FR, Otto M: Comparative analysis of virulence and toxin expression of global community-associated methicillin-resistant Staphylococcus aureus strains. J Infect Dis 2010, 202(12):1866-1876.

8. Bhakdi S, Tranum-Jensen J: Alpha-toxin of Staphylococcus aureus. Microbiol Rev 1991, 55(4):733-751.

9. Bubeck Wardenburg J, Bae T, Otto M, Deleo FR, Schneewind O: Poring over pores: alpha-hemolysin and Panton-Valentine leukocidin in Staphylococcus aureus pneumonia. Nat Med 2007, 13(12):1405-1406.

10. Kennedy AD, Bubeck Wardenburg J, Gardner DJ, Long D, Whitney AR, Braughton KR, Schneewind O, DeLeo FR: Targeting of alpha-hemolysin by active or passive immunization decreases severity of USA300 skin infection in a mouse model. $J$ Infect Dis 2010, 202(7):1050-1058

11. Wang R, Braughton KR, Kretschmer D, Bach TH, Queck SY, Li M, Kennedy AD, Dorward DW, Klebanoff SJ, Peschel A, et al: Identification of novel cytolytic peptides as key virulence determinants for community-associated MRSA. Nat Med 2007, 13(12):1510-1514.

12. Cheung GY, Duong AC, Otto M: Direct and synergistic hemolysis caused by Staphylococcus phenol-soluble modulins: implications for diagnosis and pathogenesis. Microbes Infect 2012, 14(4):380-386.

13. Coombs GW, Nimmo GR, Pearson JC, Christiansen KJ, Bell JM, Collignon PJ, McLaws ML, Resistance AGfA: Prevalence of MRSA strains among Staphylococcus aureus isolated from outpatients, 2006. Commun Dis Intell 2009, 33(1):10-20.

14. Chua KY, Seemann T, Harrison PF, Monagle S, Korman TM, Johnson PD, Coombs GW, Howden BO, Davies JK, Howden BP, et al: The dominant Australian community-acquired methicillin-resistant Staphylococcus aureus clone ST93-IV [2B] is highly virulent and genetically distinct. PLoS One 2011, 6(10):e25887.

15. Tong SY, Sharma-Kuinkel BK, Thaden JT, Whitney AR, Yang SJ, Mishra NN Rude T, Lilliebridge RA, Selim MA, Ahn SH, et al: Virulence of endemic nonpigmented northern Australian Staphylococcus aureus clone (clonal complex 75, S. argenteus) is not augmented by staphyloxanthin. J Infect Dis 2013, 208(3):520-527.

16. Labandeira-Rey M, Couzon F, Boisset S, Brown EL, Bes M, Benito Y, Barbu EM, Vazquez V, Hook M, Etienne J, et al: Staphylococcus aureus Panton-Valentine leukocidin causes necrotizing pneumonia. Science 2007, 315(5815):1130-1133.

17. Coombs GW, Goering RV, Chua KY, Monecke S, Howden BP, Stinear TP Ehricht R, O'Brien FG, Christiansen KJ: The molecular epidemiology of the highly virulent ST93 Australian community Staphylococcus aureus strain PLoS One 2012, 7(8):e43037.

18. Somerville GA, Cockayne A, Durr M, Peschel A, Otto M, Musser JM: Synthesis and deformylation of Staphylococcus aureus delta-toxin are linked to tricarboxylic acid cycle activity. J Bacterio/ 2003, 185(22):6686-6694.

19. Diep BA, Gill SR, Chang RF, Phan TH, Chen JH, Davidson MG, Lin F, Lin J, Carleton HA, Mongodin EF, et al: Complete genome sequence of USA300, an epidemic clone of community-acquired meticillin-resistant Staphylococcus aureus. Lancet 2006, 367(9512):731-739.

20. Howden BP, Stinear TP, Allen DL, Johnson PD, Ward PB, Davies JK: Genomic analysis reveals a point mutation in the two-component sensor gene graS that leads to intermediate vancomycin resistance in clinical Staphylococcus aureus. Antimicrob Agents Chemother 2008, 52(10):3755-3762.

21. Voyich JM, Otto M, Mathema B, Braughton KR, Whitney AR, Welty D, Long RD, Dorward DW, Gardner DJ, Lina G, et al: Is Panton-Valentine leukocidin the major virulence determinant in community-associated methicillin-resistant Staphylococcus aureus disease? J Infect Dis 2006, 194(12):1761-1770.

22. Bubeck Wardenburg J, Palazzolo-Ballance AM, Otto M, Schneewind O, DeLeo FR: Panton-Valentine leukocidin is not a virulence determinant in murine models of community-associated methicillin-resistant Staphylococcus aureus disease. J Infect Dis 2008, 198(8):1166-1170.

23. Chua K, Seemann T, Harrison PF, Davies JK, Coutts SJ, Chen H, Haring V, Moore R, Howden BP, Stinear TP: Complete genome sequence of Staphylococcus aureus strain JKD6159, a unique Australian clone of ST93-IV community methicillin-resistant Staphylococcus aureus. J Bacteriol 2010, 192(20):5556-5557.

24. Cue D, Lei MG, Luong TT, Kuechenmeister L, Dunman PM, O'Donnell S, Rowe S, O'Gara JP, Lee CY: Rbf promotes biofilm formation by
Staphylococcus aureus via repression of icaR, a negative regulator of icaADBC. J Bacterio/ 2009, 191(20):6363-6373.

25. Lei MG, Cue D, Roux CM, Dunman PM, Lee CY: Rsp inhibits attachment and biofilm formation by repressing fnbA in Staphylococcus aureus MW2. J Bacteriol 2011, 193(19):5231-5241.

26. Montgomery CP, Boyle-Vavra S, Daum RS: Importance of the global regulators agr and saeRS in the pathogenesis of CA-MRSA USA300 infection. PLoS One 2010, 5(12):e15177.

27. Cheung GY, Wang R, Khan BA, Sturdevant DE, Otto M: Role of the accessory gene regulator agr in community-associated methicillin-resistant Staphylococcus aureus pathogenesis. Infect Immun 2011, 79(5):1927-1935.

28. Cheung AL, Eberhardt KJ, Chung E, Yeaman MR, Sullam PM, Ramos M, Bayer AS: Diminished virulence of a sar-/agr- mutant of Staphylococcus aureus in the rabbit model of endocarditis. J Clin Invest 1994, 94(5):1815-1822.

29. Wright JS 3rd, Jin R, Novick RP: Transient interference with staphylococcal quorum sensing blocks abscess formation. Proc Natl Acad Sci U S A 2005, 102(5):1691-1696.

30. Kanehisa M, Goto S, Sato Y, Furumichi M, Tanabe M: KEGG for integration and interpretation of large-scale molecular data sets. Nucleic Acids Res 2012, 40(Database issue):D109-D114.

31. Lim Y, Jana M, Luong TT, Lee CY: Control of glucose- and NaCl-induced biofilm formation by rbf in Staphylococcus aureus. J Bacteriol 2004, 186(3):722-729.

32. Rasband WS: ImageJ. Bethesda, Maryland, USA: U S National Institutes of Health available at http:/imagej.nih.gov/ij/, accessed 9 December 2009 1997-2011.

33. Nguyen HM, Rocha MA, Chintalacharuvu KR, Beenhouwer DO: Detection and quantification of Panton-Valentine leukocidin in Staphylococcus aureus cultures by ELISA and Western blotting: diethylpyrocarbonate inhibits binding of protein A to IgG. J Immunol Methods 2010, 356(1-2):1-5.

34. Bae T, Schneewind O: Allelic replacement in Staphylococcus aureus with inducible counter-selection. Plasmid 2006, 55(1):58-63.

35. Monk IR, Shah IM, Xu M, Tan MW, Foster TJ: Transforming the untransformable: application of direct transformation to manipulate genetically Staphylococcus aureus and Staphylococcus epidermidis. MBio 2012, 3(2):e00277-00211.

36. Li MZ, Elledge SJ: Harnessing homologous recombination in vitro to generate recombinant DNA via SLIC. Nat Methods 2007, 4(3):251-256.

37. Howden BP, McEvoy CR, Allen DL, Chua K, Gao W, Harrison PF, Bell J, Coombs G, Bennett-Wood V, Porter $J$, et al: Evolution of multidrug resistance during Staphylococcus aureus infection involves mutation of the essential two component regulator WalKR. PLOS Pathog 2011, 7(11):e1002359.

38. Rumble SM, Lacroute P, Dalca AV, Fiume M, Sidow A, Brudno M: SHRiMP: accurate mapping of short color-space reads. PLoS Comput Biol 2009, 5(5):e1000386

39. David M, Dzamba M, Lister D, llie L, Brudno M: SHRiMP2: sensitive yet practical SHort Read Mapping. Bioinformatics 2011, 27(7):1011-1012.

40. Robinson MD, McCarthy DJ, Smyth GK: edgeR: a Bioconductor package for differential expression analysis of digital gene expression data. Bioinformatics 2010, 26(1):139-140.

doi:10.1186/1471-2180-14-31

Cite this article as: Chua et al:: Hyperexpression of a-hemolysin explains enhanced virulence of sequence type 93 community-associated methicillin-resistant Staphylococcus aureus. BMC Microbiology 2014 14:31.

\section{Submit your next manuscript to BioMed Central and take full advantage of:}

- Convenient online submission

- Thorough peer review

- No space constraints or color figure charges

- Immediate publication on acceptance

- Inclusion in PubMed, CAS, Scopus and Google Scholar

- Research which is freely available for redistribution 